\title{
Predicción política y Twitter: Elecciones generales de España 2015
}

\author{
Iragarpen politikoa eta Twitter: 2015eko \\ Espainiako hauteskunde orokorrak
}

\author{
Political prediction and Twitter: \\ Spanish legislative elections 2015
}

\section{Marián Alonso González'}

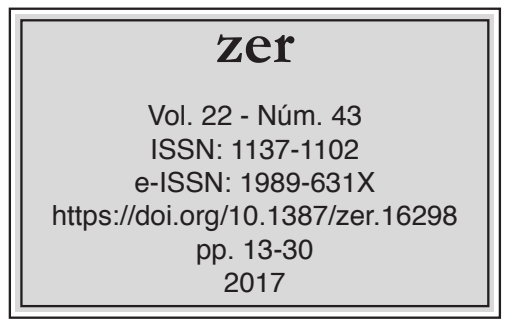

Recibido el 5 de mayo de 2016, aceptado el 2 de julio de 2017.

\section{Resumen}

Este trabajo discute la utilidad y alcance del uso de las redes sociales durante las campañas electorales. Se presentan los resultados de una investigación que analiza el empleo de Twitter por los partidos políticos en las elecciones generales de España del 20 de diciembre de 2015. Para ello se cuantifica la presencia de partidos y candidatos durante el último mes de campaña, y se analiza la cantidad y calidad de sus interacciones. Los resultados indican que los datos extraídos de Twitter son un buen termómetro social pero que aún tiene por delante un importante reto metodológico como herramienta predictiva.

Palabras clave: Opinión pública, Web 2.0, Twitter, partidos políticos, elecciones.

Hauteskunde kanpainetan sare sozialen erabilerak duen baliagarritasuna eta helmena eztabaidagai du azterlan honek. 2015eko abenduaren 20ko Espainiako hauteskunde orokorretan alderdi politikoek Twitter nola erabili zuten aztertzen duen ikerlan baten emaitzak azaltzen ditu. Ikerlan horrek kanpainaren azken hilabetean alderdien eta hautagaien agerpenak kuantifikatu ditu, eta interakzioen kopurua eta kalitatea ere aztertu ditu. Emaitzek adierazten dute Twitterretik ateratako datuak termometro sozial onak direla, baina iragarpen-tresna izateko erronka metodologiko handiak gainditu beharko dituela.

Gako-hitzak: Iritzi publikoa, Web 2.0, Twitter, alderdi politikoak, hauteskundeak.

\footnotetext{
1 Universidad de Sevilla, malonsog@us.es
} 


\begin{abstract}
This paper discussed the utility and reach of social networks during electoral campaigns. We presented the results of a research that analyzes the use of Twitter by political parties in the legislative elections in Spain December 20 ${ }^{\text {th }}, 2015$. Presence of the candidates and political parties during the last month of campaign was quantified, and the quality and quantity of their interactions with their followers was evaluated. The results indicate that Twitter is a good social thermometer, but still it faces a major methodological challenge as a predictive tool.
\end{abstract}

Keywords: Public opinion, Web 2.0, Twitter, political parties, elections. 


\section{Introducción}

El análisis de campañas electorales online ha puesto de manifiesto que la red tiene capacidad de influir de forma positiva en los resultados electorales y que los candidatos con mejor posicionamiento en los medios sociales pueden aventajar a sus oponentes.

Desde que Barack Obama obtuviese la presidencia estadounidense en 2008 gracias a su "Yes we can", y a una decida apuesta por internet como punto clave de su campaña electoral, ha quedado más que patente la convicción de que cualquier candidato que quiera conseguir una mejoría en sus resultados, e incluso el éxito electoral, está prácticamente obligado a desarrollar una cibercampaña.

El interés por conocer la opinión pública tiene una amplia tradición como campo de estudio, especialmente cuando se relaciona con el concepto de democracia y se erige en uno de los principales baluartes de la comunicación política. Con tal fin se han desarrollado numerosas técnicas de investigación que permiten obtener una información representativa de la opinión de los individuos, entre ellas las tradicionales encuestas demoscópicas de intención de voto. Sin embargo, la necesidad de descifrar la capacidad que las plataformas 2.0 poseen para articular corrientes de opinión transnacionales pone de manifiesto una necesidad de cambio metodológico en los estudios de opinión pública y explica el surgimiento de los análisis digitales.

Existe un nuevo universo opinativo que es digital. Los medios sociales han contribuido a democratizar la información generando un proceso comunicativo bidireccional que cambia de forma significativa la participación política, ya que se convierten en una ágora virtual donde el ciudadano puede exponer problemáticas no recogidas en la agenda pública y mostrar sus opiniones de una manera libre.

A lo largo de este artículo proponemos, a través del estudio de los mensajes que los seis principales partidos políticos españoles (PP, PSOE, IU, UPyD, Ciudadanos y Podemos) publicaron en Twitter, establecer una correlación entre la actividad desarrollada en este medio social y los resultados electorales, a fin de comprobar que Twitter puede llegar a ser una herramienta predictiva y corroborar que las conclusiones publicadas por otros investigadores y analistas políticos en otros países son aplicables a España.

Nuestro interés por esta red social, frente a otras posibles opciones, es debido a su carácter abierto al público, cuya capacidad de irradiación no depende solo "de los seguidores que tenga una cuenta determinada, sino del alcance exponencial de los mensajes redifundidos por dichos seguidores" (López-García, 2016: 151).

Twitter permite marcar la agenda y establecer cuáles son los mensajes prioritarios para el emisor, así como su orden de aparición y la naturaleza de los mismos, también se corresponde con precisión con la realidad comunicativa propiciada por el nuevo escenario, caracterizada por acortamiento de los ciclos de noticias y su difusión fragmentaria, a menudo, descontextualizada entre el público (Barber, 2004: 38).

Frente a la legitimidad de las democracias representativas tradicionales en las que se otorga un gran poder a la agenda-setting, entendida como la alianza tácita que existe entre el gobierno de un país y los medios de comunicación para informar a los espectadores, oyentes o lectores de un determinado medio sólo lo que interesa (Monge, 2011), los medios sociales abren un espacio digital que garantiza la participación, 
"una oportunidad para la ciudadanía, en términos de activismo y deliberación sobre los asuntos públicos" (Resina, 2010: 143).

Se genera, pues, un ecosistema comunicativo que Chadwick (2013: 44) denomina "híbrido", pues en él conviven viejos y nuevos medios, los cuales se retroalimentan unos de otros, en el que el público continúa consumiendo medios tradicionales, pero con rutinas de consumo distintas a las habituales (Jenkins, 2008) y en el que los ciclos de noticias se acortan o intensifican y cuyo recorrido se extiende y tiene derivaciones en internet y las redes sociales. En este sistema, las principales instituciones mediáticas continúan siendo las que ya lo eran en el modelo anterior, es decir, los grandes medios continúan marcando la agenda y condensando la atención del público y la influencia social, sin embargo, "lo hace generando versiones digitales de medios convencionales que operan en internet ofreciendo contenidos en esencia similares a los del medio fuente" (Chadwick, 2013:88).

Si bien es cierto que los grandes medios siguen ocupando una posición central, las redes sociales ponen de manifiesto que existe una alternativa mediática, pues "un grupo de personas, no necesariamente conectadas entre sí, puede contrastar una noticia a una velocidad superior de lo que podría hacerlo un simple periodista" (RosMartin, 2005: 1). Dentro de este contexto, la política se enfrenta a una realidad en la que internet y los medios sociales brindan a los ciudadanos el poder de tener una red asociativa capaz de provocar los cambios que los políticos no llevan a cabo y, es por ello, que fija sus objetivos en el terreno de las herramientas 2.0 como medio de acercamiento a la ciudadanía.

Las herramientas digitales crean grupos de pertenencia y corrientes de opinión ampliamente aceptadas debido a dos mecanismos convergentes: el efecto cascada y la polarización de grupos (Sunstein, 2009). El efecto cascada es aquel por el que la señal se refuerza cuanta más gente la recibe, hasta llegar a un punto en que es casi imposible resistirse a ella. Por su parte, la polarización de grupos es una forma de asimilación tendenciosa de la información porque proviene de personas de mentalidad afín o con intereses compartidos. Es decir, "nuestras opiniones se fortalecen y hacen más extremas cuando las compartimos con personas afines y estas nos las corroboran, pero también cuando las discutimos con quienes discrepamos afianzándonos de nuevo en su error y en nuestro acierto" (Alonso, 2015: 397).

La combinación de ambos factores contribuye al desarrollo de una causalidad recíproca asimétrica cuyas evidencias han sido encontradas en aspectos relacionados con la eficacia política (Semetko \& Valkenburg, 1998), la participación cívica (Shah et al., 2005) y la participación política (Rojas, 2006). En este sentido, la conversación política entre ciudadanos contribuye a la creación de una polis activa y comprometida que genera una mayor tolerancia cuando la conversación sucede en redes sociales heterogéneas (Mutz, 2002).

Según Maarek (2014), los medios digitales han sido incorporados como herramientas de comunicación política por tres razones principales. La primera es la velocidad, dado que cualquier mensaje político puede ser subido desde cualquier lugar del mundo y casi instantáneamente descargado en cualquier otro sitio, sin demora, debido a las potentes capacidades de los motores de búsqueda como Google o Yahoo. La segunda razón es la versatilidad. Los medios digitales admiten textos en sitios web (websites) y en blogs, mensajes públicos y privados, fotos y vídeos. 
Finalmente, la tercera razón es su facilidad de uso: la comunicación política ya no es tan sólo de arriba hacia abajo (top-down), de los partidos y los medios hacia los ciudadanos, sino que ahora es también horizontal y de abajo hacia arriba (bottomup), de forma que cualquier persona digitalmente conectada puede convertirse en fuente de comunicación.

Hasta que Obama llegó a la Casa Blanca, las redes sociales sólo se habían mostrado como poderosas herramientas de convocatoria y recolección de fondos. Sin embargo, el equipo de campaña del presidente norteamericano puso de manifiesto que los medios sociales eran unos importantes instrumentos para alcanzar y organizar a los votantes, como foro de debate y como espacios de creación de liderazgos virtuales y personales.

Los medios sociales permiten viralizar las campañas convencionales, estimulan la participación, permiten que los ciudadanos aporten sus puntos de vista y sus críticas; dando origen a "narrativas políticas transmedia" (D'Adamo et al., 2015: 109), que ofrecen la posibilidad de expandir el mensaje o la campaña, en este caso, por varios medios, junto con la colaboración del ciudadano votante en ese proceso expansivo. Asimismo, otorgan la posibilidad de dar una respuesta rápida a los ataques de los opositores y poseen capacidad de influencia en la agenda de los medios convencionales. De hecho, los periódicos, sobre todo en su edición online, suelen nutrirse de los tuits de los políticos, y cuando los reproducen, amplifican su difusión, que llega así a un público más extenso que el de los seguidores.

\section{Predicción política y Twitter}

La creciente importancia de los medios digitales en las campañas electorales reside en el nacimiento de una Comunicación Política 2.0 en la que la interacción es clave, ya que los ciudadanos necesitan sentir que forman parte de un proyecto político compartido, y para empatizar con ellos, dice Herrero (2014), nada mejor que incorporar al mensaje político aquellos temas de la agenda pública que más preocupan a los ciudadanos.

A través de la escucha y la monitorización de los temas más relevantes, los medios sociales llegan a establecer un control sobre las conversaciones, lo que derivará en la puesta en marcha de "acciones referentes a la creación de contenido, participación, interactividad y, sobre todo, la construcción de relaciones" (Leiva-Aguilera, 2012: 31). De hecho, los estudios de García y Zugasti (2014) y Zugasti y Sabés (2015) aprecian que el interés de los candidatos electorales por responder a los ciudadanos que les interpelan en Twitter tiene como efecto colateral una mayor diversidad temática por parte del candidato, y que de ahí saldría el argumentario y agenda temática que presidirá la estrategia de campaña.

Por todo ello, la presencia de partidos y políticos en las redes sociales se ha convertido en un desafío en términos teóricos, hasta el punto de que ya ha comenzado a ser investigado por la academia (Giansante, 2015). En este sentido, son muchos los estudios científicos orientados a entender los modelos de predicción política por medio de las redes sociales y, más concretamente, a través de Twitter por tratarse de una red extendida y reconocida como medio de expresión y difusión de opiniones, pero también por la facilidad que ofrece para capturar dichos datos gracias a su public time-line. 
Así, por ejemplo, encontramos el trabajo realizado durante las elecciones de 2009 en Alemania por Tumasjan et al. (2010) quienes formulan una teoría que parece demostrar que el número de usuarios de Twitter y sus mensajes podían explicar la tendencia de voto. Estos autores se basan en estudios anteriores y similares como el de Véronis (2007) sobre las elecciones presidenciales de 2007 en Francia, en el que se afirma que el número de apariciones o de menciones de un candidato en la prensa puede ser un buen predictor del resultado de la contienda.

También en 2010, O’Connor et al. relacionan la opinión pública en EEUU en el periodo 2008-2009 a través de una comparativa entre las encuestas tradicionales y el sentimiento reflejado en los tuits. Sus resultados fueron coincidentes en casi el $80 \%$, no obstante, los autores sugieren mejorar las herramientas de PNL (programación neurolingüística) para enriquecer dichas estimaciones.

En esta misma línea, Larsson y Moe (2010), tras estudiar las elecciones de 2010 en Suecia, afirman que el manejo de los mensajes de Twitter es más complejo de lo pensado y que requiere una atención especial. En ambos estudios se hace un serio hincapié en la tendencia y en la predicción.

El uso de Twitter y las redes sociales en las campañas políticas son investigados en el caso de las elecciones en Rumanía y España por Aparashivei (2011) y Deltell et al. (2013), respectivamente. En una línea parecida, Al-Kandari y Hasanen (2012) estudian el impacto que los medios sociales poseen en las decisiones políticas en Egipto y Kuwait.

Si algo tienen en común todos los estudios mencionados es que, aplicando técnicas diferentes en distintos momentos sobre los datos capturados en Twitter, arrojan resultados positivos en lo que se refiere a la capacidad de utilizar este medio social como un método de predicción electoral que sustituya y se combine con las encuestas tradicionales.

Los diferentes estudios ponen de manifiesto que los medios sociales se revelan como un campo nuevo para la investigación en estudios dedicados a analizar la influencia de las campañas electorales en la decisión del voto de los individuos. En este sentido, las elecciones generales de 2010 de Reino Unido fueron objeto de estudio pocos días después de la celebración de los comicios por parte de la plataforma de medios Tweetminster (2010).

Con una metodología similar a la empleada por Tumasjan et al. (2010) (recolectando tuits mediante el API de Twitter que menciona a un candidato y sumando las menciones obtenidas por cada candidato) llegaron a la conclusión de que el candidato con más menciones resultó ser el vencedor de la contienda, de forma que a mayor volumen de datos, mayor grado de acierto; y que "tanto el éxito en el acierto, como el porcentaje de desviación de la predicción de los resultados electorales, es comparable al obtenido por las encuestas" (Tweetminster, 2010: 6).

Sin embargo, la sola presencia de un candidato en internet o de desarrollar una excelente campaña de comunicación online, no es sinónimo de victoria electoral, ya que, existen otros muchos factores que influyen como la imagen, credibilidad y liderazgo del aspirante, el clima social y la oportunidad de conseguirlo, o el éxito de las campañas físicas que se desarrollan a la par. Prueba de ello es que frente al éxito arrollador que supuso la campaña online para Obama encontramos otros casos en los que el preferido de las redes sociales no resultó el vencedor electoral, es el caso de 
Antanas Mockus, candidato por el Partido Verde de Colombia, y que fue derrotado por su oponente, Juan Manuel Santos.

Ello ha generado toda una corriente de investigación crítica con la capacidad de predicción de resultados electorales por parte de Twitter. Así, Jungherr et al. (2012) cuestionan el método empleado por Tumasjan et al. para la elección del período de estudio, además de indicar que el número de menciones en la globosfera no podía utilizarse como un indicador.

También Tjong y Bos (2012) cuestionaron si contando simplemente las menciones a los partidos políticos en Twitter se podía predecir el resultado de las elecciones al Senado holandés en 2011. Ambos autores valoraron el sentimiento de los tuits, si eran positivos o negativos hacia el partido en cuestión. Otros estudios y autores, como Gayo-Avello y Panagiotis (2011), llegan incluso a mencionar la falacia del "big data", es decir, que el hecho de obtener grandes muestras y colecciones estadísticas no hace que éstas sean necesariamente representativas de la población.

En España también contamos con varios trabajos relacionados con el uso de Twitter en las campañas electorales. Así, Zamora y Zurutuza (2014) sobre las elecciones generales de 2011 llegan a la conclusión de que los candidatos presidenciales replican en esta red social las características del discurso político tradicional: unilateral y sin interacción, una conclusión similar a la de Borondo et al. (2012) quienes afirman que los candidatos ven en los medios sociales herramientas de las que intentan beneficiarse para ganar votos, pero no como un auténtico canal de comunicación. Olvidan, no obstante, estos estudios el gran potencial de movilización y activismo que poseen las redes, en especial para públicos como los jóvenes (García et al., 2014), objetivo de toda campaña electoral debido a su dificultad de movilización en estos procesos.

Twitter no sólo es hablar, es escuchar, responder y debatir, y este potencial que permite romper barreras sin tener que contar con intermediarios (Rodríguez y Ureña, 2011) constata que las redes sociales comienzan a compartir el espacio de los cibermedios de comunicación como narradores de la realidad (García et al., 2012), tareas hasta hace poco limitadas, exclusivamente, al ámbito del periodismo tradicional, convirtiendo en tendencia o "trending topic" asuntos que no figuran entre los más destacados en los medios de comunicación clásicos (Martins y Azevedo, 2015).

\section{Objetivos y metodología}

Twitter está contribuyendo a transformar y redefinir el activismo político, abriendo nuevas oportunidades y escenarios a los movimientos sociales en cuanto a su organización y movilización y reforzando el papel de la comunicación política (Casero, 2015) pero, además, su incorporación al ámbito de la comunicación empresarial e institucional ha puesto de manifiesto que se trata de una herramienta muy útil a la hora de hacer de la comunicación un ámbito ágil, transversal y no lineal, lo que permite amplificar el mensaje, aumentar la visibilidad de las corporaciones y, por ende, facilitar la difusión de información.

En base a ello, y siguiendo la estela de los trabajos anteriormente mencionados, la hipótesis de partida de este artículo es que la comunicación a través de Twitter permite aumentar la visibilidad de partidos y políticos, erigiéndose en importantes instrumentos para comunicarse con los electores y dar a conocer sus programas 
electorales, lo cual puede traducirse en un mayor número de votos. En este sentido, nuestra hipótesis central es que Twitter sirve para predecir la tendencia electoral. Sin embargo, nuestro trabajo también explorará las nuevas formas de liderazgo en Twitter como presentan Said y Arcila (2011), la creación de juegos y roles políticos como observa Wilson (2011) en Australia y el alcance de Twitter como herramienta política, descrito por González y Petersen (2010) en Venezuela.

Siguiendo a Delttel et al. (2013), este trabajo se sitúa en un punto intermedio entre la comunicación vertical de las organizaciones políticas tradicionales y la "autocomunicación de masas" definida por Castells (2009), es por ello que para verificar nuestra hipótesis el presente estudio tiene como objetivo analizar las tasas de conversación, amplificación y gratificación que los seis principales partidos políticos españoles (PP, PSOE, IU, UPyD, Ciudadanos y Podemos) desarrollaron en Twitter durante la campaña electoral de 2015 en España para aclarar el potencial de influencia que poseen en sus seguidores e intentar establecer una correlación entre la actividad desarrollada en esta plataforma y los resultados electorales.

A fin de comprobar que Twitter es una herramienta predictiva y corroborar que las conclusiones publicadas por otros investigadores y analistas políticos en otros países son aplicables a España, recurrimos a una metodología mixta cuyas ventajas han sido destacadas por autores como Creswell y Plano (2011) en respuesta a la oposición dicotómica entre ambos planteamientos, pues permite una comprensión del problema de investigación en el cual se articula la utilización de estadísticas y la medición de determinados fenómenos, propios de lo cuantitativo, con la exploración en profundidad de la problemática examinada y la extracción y comprensión de los significados vinculados las mismas, característico de lo cualitativo.

La aplicación sistemática de ambos modelos nos permite una acumulación ordenada y estructurada de datos que nos ha facilitado la extracción de inferencias de interés para los objetivos de esta investigación.

En total, hemos estudiado 13.831 mensajes que fueron publicados entre el 17 de noviembre y el 17 de diciembre de 2015 . Se trata del mes previo a la jornada electoral a fin de dotar de mayor actualidad al estudio y por considerar que se trata de un plazo idóneo para poder desarrollar un correcto estudio longitudinal.

Los datos obtenidos en Twitter los hemos categorizado en torno a 12 indicadores (presencia, tuits totales, seguidores, siguiendo, seguidores/siguiendo, listas, tuits emitidos durante el periodo de estudio, favoritos, retuits, respuestas, menciones y enlaces multimedia) agrupados en 5 apartados: presencia, potencial de influencia, actividad, interacción y reacciones generadas en los usuarios, siguiendo la ficha de análisis propuesta por Pérez-Dasilva et al. (2015). Además, para calcular el engagement hemos recurrido a la herramienta Tweetstat que funciona en base a algoritmos que aseguran la validez científica de los datos obtenido con niveles de confianza que no bajan de $95 \%$ y un error muestral inferior al $+/-5 \%$.

A nivel de contenido hemos categorizado los mensajes analizados en torno a los ocho temas más recurrentes en sus discursos y que son: Empleo, Economía, Sociedad, Sanidad, Política, Corrupción, Educación y Otros, al tiempo que hemos otorgado un valor positivo, negativo o neutro a los mensajes publicados. Con ello pretendemos realizar un perfil de los temas en los que concentran su interés, cuáles son las principales funciones para las que emplean esta red social: difundir mensajes políticos, 
actos de campaña o enfatizar aspectos personales; así como establecer una correlación entre sus discursos online y los resultados arrojados por el sondeo de opinión realizado por el Centro de Investigaciones Sociológicas (CIS) de diciembre de 2015.

\section{Resultados}

Según el estudio de intención de voto realizado por el Centro de Investigaciones Sociológicas (CIS) para los comicios electorales de 2015, el PP se alzaría con la victoria con un $28,6 \%$ de intención de voto y entre 120 y 128 escaños. La macroencuesta electoral daba al PSOE entre 77 y 79 escaños (20,8\% de votos), seguido de Ciudadanos (19\% y entre 63 y 66 escaños) y por Podemos que, junto a otras marcas de la formación, podría llegar al 15,7\% de los votos y conseguir hasta 49 diputados. La encuesta del CIS también otorgaba a IU, entre 3 y 4 escaños (3,6\% de los votos) y un escaso $0,7 \%$ a UPyD que dejaría sin presencia en el Congreso de los Diputados a la formación magenta.

Las encuestas de opinión sobre expectativa de voto suelen ser un recurso muy habitual en las campañas electorales, aunque no siempre sus predicciones se ajustan a los resultados electorales debido a que se realizan en un instante dado y con un conjunto de preguntas concretas, es por ello que los datos ofrecidos por el CIS erraban en el caso de las nuevas formaciones, ya que Podemos llegó al 20,66\% de los votos, mientras que Ciudadanos se quedaba con sólo el 13,93\%, como puede apreciarse en la siguiente tabla.

Tabla 1. Comparativa de resultados y encuestas Elecciones 20D.

\begin{tabular}{|l|c|c|}
\hline & Resultados 20D & Datos CIS \\
\hline PP & $28,72 \%$ & $28,60 \%$ \\
\hline PSOE & $22,02 \%$ & $20,80 \%$ \\
\hline Podemos & $20,66 \%$ & $15,70 \%$ \\
\hline IU & $3,67 \%$ & $3,60 \%$ \\
\hline Ciudadanos & $13,93 \%$ & $19 \%$ \\
\hline UPyD & $0,61 \%$ & 0,7 \\
\hline
\end{tabular}

Fuente: Elaboración propia.

Para superar las limitaciones de los sondeos, el presente trabajo parte de la base de que Twitter actúa como una sonda y, por tanto, podría permitir medir la opinión de forma continuada en un periodo más largo, ya que los tuits emitidos con opinión política supera a las encuestas recogidas por cualquier sondeo, de manera que sería posible establecer una relación entre el uso de Twitter y los resultados electorales finalmente cosechados por las formaciones políticas.

En este sentido, el presente trabajo propone un sistema de predicción que consta de dos partes: una cuantitativa basada en el análisis del número de seguidores de los perfiles de los partidos políticos; y una segunda basada en el uso de herramientas de minería de opinión para calcular el impacto de los tuits y de los líderes de opinión 
que se crean en torno al flujo de mensajes, así como la carga negativa, positiva o neutra del tuit en ese contexto.

\subsection{Análisis cuantitativo}

Izquierda Unida fue el primer partido en abrir perfil en esta red social, en mayo de 2008. Con posterioridad, en 2009, lo hacen Ciudadanos (enero), Partido Popular (febrero), PSOE (junio). Los más recientes son UPyD (mayo de 2010) y Podemos (marzo de 2014). No obstante, son@ @ahorapodemos y @Ppopular los que poseen un mayor potencial de influencia, lo que pone de manifiesto que la popularidad en Twitter no está ligada de forma sistemática con la antigüedad en esta red social. Ambas formaciones también lideran tanto en número de usuarios como en la métrica seguidores/seguidos.

Tabla 2. Potencial de influencia.

\begin{tabular}{|l|r|r|r|r|}
\hline & \multicolumn{1}{|c|}{ Siguiendo } & Seguidores & Seg/sig & \multicolumn{1}{c|}{ Listas } \\
\hline @ahorapodemos & 1.120 & 835.388 & 745 & 2 \\
\hline @PPopular & 2.530 & 418.854 & 165 & 12 \\
\hline @PSOE & 8.630 & 327.945 & 38 & 56 \\
\hline @iunida & 4.088 & 270.867 & 66 & 11 \\
\hline @UPyD & 1.123 & 166.897 & 148 & 19 \\
\hline @CiudadanosCs & 79.285 & 251.858 & 3 & 9 \\
\hline
\end{tabular}

Fuente: Elaboración propia.

A nivel cuantitativo nos hemos basado en el trabajo de Tumasjan et al. (2010), para realizar una predicción de voto mediante Twitter y por el cual el número de seguidores resulta relevante y marca una tendencia de voto. En esta línea, para obtener un método de predicción promediamos el tanto por ciento de los seguidores de los partidos políticos con el de los cabezas de lista y el resultado ofrecería la predicción de votos en tanto por ciento (Ver Tabla 3).

Tabla 3. Seguidores en Twitter de partidos y líderes políticos.

\begin{tabular}{|l|c|l|c|c|}
\hline \multicolumn{1}{|c|}{ Partido } & Seguidores & \multicolumn{1}{c|}{ Candidato } & Seguidores & Promedio \\
\hline @ahorapodemos & 835.388 & @Pablo_Iglesias_ & 1.380 .642 & $34,4 \%$ \\
\hline @PPopular & 418.854 & @marianorajoy & 1.020 .654 & $22,3 \%$ \\
\hline @PSOE & 327.945 & @sanchezcastejon & 224.019 & $8,5 \%$ \\
\hline @iunida & 270.867 & @agarzon & 432.979 & $10,9 \%$ \\
\hline @UPyD & 166.897 & @Herzogoff & 13.500 & $2,8 \%$ \\
\hline @CiudadanosCs & 251.858 & @Albert_Rivera & 431.553 & $10,60 \%$ \\
\hline
\end{tabular}

Fuente: Elaboración Propia (2015)-

$\mathrm{Al}$ realizar una comparativa de las previsiones obtenidas por nuestro estudio y los resultados obtenidos el 20-D hemos tenido en cuenta que entre las seis formaciones 
tuvieron el $89,61 \%$ de los votos, porcentaje que aplicamos a la hora de calcular los resultados electorales en base al número de seguidores que posee un partido y el candidato. No obstante, como podemos comprobar en la Tabla 4 esta métrica no resulta relevante a la hora de marcar la tendencia de voto, de hecho, apenas se aproxima a los resultados finales pues, según la misma, Podemos obtendría el 34,4\% de los votos, seguido del 22,3\% del PP y el 10,9\% de IU, cuando la realidad es que el PP obtuvo el 28,7\% de los votos, Podemos se quedó en el 20,6\% e IU sólo logró el 3,67\%.

Tabla 4. Comparativa de resultados y previsiones en Twitter.

\begin{tabular}{|l|c|c|}
\hline \multirow{2}{*}{} & \multirow{2}{*}{ Resultados 20D } & Previsión \\
\cline { 3 - 3 } & & redes sociales \\
\hline PP & $28,72 \%$ & $22,3 \%$ \\
\hline PSOE & $22,02 \%$ & $8,5 \%$ \\
\hline IU & $20,66 \%$ & $34,4 \%$ \\
\hline Ciudadanos & $3,67 \%$ & $10,9 \%$ \\
\hline UPyD & $13,93 \%$ & $10,6 \%$ \\
\hline
\end{tabular}

A fin de superar las singularidades y fallas técnicas que no permiten un modelo de predicción global, algunos autores como Cavaller et al. (2013) proponen calcular el engagement. A nivel de marketing, el engagement se podría definir como el establecimiento de una relación con un cliente potencial, lo cual, a lo largo del tiempo puede desembocar en una compra. A nivel político, no buscaríamos la compra de un producto, pero sí el voto, es por ello, que uno de los objetivos de la comunicación en las redes sociales es estrechar lazos e ir realizando un acercamiento a los votantes potenciales para que se conciencien de los valores que representa la formación política y cale con mayor profundidad las consignas lanzadas.

En este sentido, y con objeto de establecer la capacidad que tienen los partidos de influir en su comunidad de seguidores hemos analizado las métricas correspondientes a retuits, menciones, respuestas o "quiénes te han retuiteado", todas ellas nos permiten concretar el nivel de compromiso (engagement) y de amplificación del mensaje.

Si atendemos a los retuits, todos los partidos lo hacen con asiduidad, sin embargo, son Podemos (87\%) y PSOE (86\%) los que lo hacen con gran frecuencia. En cuanto a la naturaleza de dichos retuits, la inmensa mayoría están relacionados con las cuentas del candidato y de sus primeros espadas y sirven, generalmente, para repetir mensajes relacionados con su programa político, eslóganes y actos de campaña, así como para difundir la participación de los candidatos en los medios de comunicación, de forma que el retuit se utiliza a modo de promoción y de vertebración interna de la formación.

En el ámbito de las respuestas sobresalen Partido Popular (49\%) y Podemos (37\%) que de esta forma le toman el pulso a sus seguidores para incorporar a la agenda pública los temas de mayor interés, además, en el caso de Podemos, usan 
Twitter para transmitir mensajes horizontales y como un instrumento de llamada al debate. Ambos partidos, son, además, con un $23 \%$ y un $28 \%$ respectivamente, los que de forma más evidente apuestan por incluir enlaces en sus mensajes, apostando por las ventajas hipermedia que ofrecen estos medios (Ver Tabla 5).

En el ámbito de las menciones, como vía de interacción con los usuarios, destacan las dos formaciones más jóvenes, seguidas del PP. No obstante, la naturaleza de las mismas son distintas, mientras que en Ciudadanos la interacción se produce con usuarios que aceptan o critican sus propuestas, las de Iglesias y Rajoy están orientadas a otros miembros de su partido, de forma que se usan, sobre todo, a modo de comunicación interna.

Por debajo del $10 \%$ de interacción encontramos a UPyD, el cual desarrolla un modelo comunicativo jerarquizado y vertical (towdown), usando Twitter como una plataforma complementaria para la difusión unidireccional de mensajes y desaprovechando las peculiaridades proactivas que presenta esta red social.

Tabla 5. Interacción y reacciones de los partidos en Twitter.

\begin{tabular}{|l|c|c|c|c|c|c|}
\hline & $\begin{array}{c}\text { Favoritos } \\
\%\end{array}$ & $\begin{array}{c}\text { Retuits } \\
\%\end{array}$ & $\begin{array}{c}\text { Respuestas } \\
\%\end{array}$ & $\begin{array}{c}\text { Reacción } \\
\%\end{array}$ & $\begin{array}{c}\text { Hipermedia } \\
\%\end{array}$ & $\begin{array}{c}\text { Menciones } \\
\%\end{array}$ \\
\hline @ahorapodemos & $49,51 \%$ & $87 \%$ & $37 \%$ & $57,84 \%$ & $28 \%$ & $23,18 \%$ \\
\hline @PPopular & $89,50 \%$ & $75 \%$ & $49 \%$ & $71,17 \%$ & $23 \%$ & $19,8 \%$ \\
\hline @PSOE & $53,64 \%$ & $86 \%$ & $32 \%$ & $57,21 \%$ & $16 \%$ & $18,5 \%$ \\
\hline @iunida & $11,90 \%$ & $55,23 \%$ & $29,06 \%$ & $32,06 \%$ & $9 \%$ & $8,23 \%$ \\
\hline @UPyD & $21,90 \%$ & $45,67 \%$ & $6,67 \%$ & $24,75 \%$ & $7 \%$ & $7,55 \%$ \\
\hline @CiudadanosCs & $23,45 \%$ & $57,86 \%$ & $24,08 \%$ & $35,13 \%$ & $14 \%$ & $22,5 \%$ \\
\hline \multicolumn{7}{|r|}{}
\end{tabular}

Para lograr la eficacia de una cuenta en Twitter no sólo hay que establecer un adecuado flujo comunicativo con los seguidores, también es importante conseguir una masa crítica de seguidores interesados en los mismos y, en este sentido, cobran gran relevancia los retuits y favoritos que generan.

Una cuenta con muchos mensajes reenviados por terceros es un signo inequívoco de que es considerada como una fuente de información a tener en cuenta por los usuarios. En este sentido, Podemos (87\%) y PSOE (86\%) son los partidos cuyos mensajes son más viralizados por parte de sus usuarios, aunque debemos precisar que, en general, los mensajes emitidos por los partidos políticos gozan de gran repercusión entre sus usuarios, con niveles superiores al $55 \%$.

Para conocer la tasa de gratificación hemos analizado los favoritos que consigue cada mensaje publicado. PP $(89,5 \%)$ y PSOE $(53,64 \%)$ son los partidos que registran un mayor porcentaje en la medición de esta variable, con valores superiores al $50 \%$ de sus tuits, también Podemos, con valores cercanos al 50\%, registra un buen nivel.

Promediando las métricas de respuestas, favoritos, menciones y retuits obtenemos que el PP, con un $71,17 \%$, es el partido que mayor reacción genera en sus usuarios. Le sigue Podemos $(57,84 \%)$, PSOE $(57,21 \%)$, Ciudadanos $(35,13 \%)$, IU $(32,06 \%)$ y UPyD $(24,75 \%)$.

Con objeto de porcentualizar la reacción que la comunicación en Twitter genera en los usuarios y, de esta forma, establecer una correspondencia entre actividad social 
e intención de voto, hemos recurrido a Tweetstat, una herramienta que nos permite calcular el engagement en base a una sencilla fórmula en la que suma el número total de menciones, retutis y likes y lo divide entre el número total de tuits. La cantidad resultante la divide, con posterioridad, por el número de seguidores totales y lo multiplica por 100 obteniendo un valor básico que nos sirve como punto de referencia.

Como puede apreciarse en la Tabla 6, el engagement nos permite porcentualizar el sentimiento generado por los usuarios en la red social Twitter, ofreciendo unas previsiones bastante acertadas y próximas a los resultados electorales, al menos en caso de las formaciones más jóvenes y que concurrían por primera vez a las elecciones generales.

Su actividad comunicativa otorga a Podemos el 21,4\% de los votos, mientras que a Ciudadanos le da un $13,7 \%$, porcentajes muy similares a los obtenidos en el recuento oficial $(20,7 \%$ y $13,9 \%$, respectivamente).

En el caso de PP y PSOE los datos obtenidos también se acercan bastante al porcentaje real de votos, incluso obtenemos un resultado similar al de las encuestas tradicionales en el caso de la formación socialista (22\% y 20,9\%, respectivamente).

Tabla 6. Comparativa de resultados y previsiones Engagement Twitter.

\begin{tabular}{|l|c|c|}
\hline & Resultados 20D & Engagement Twitter \\
\hline PP & $28,7 \%$ & $25,7 \%$ \\
\hline PSOE & $22 \%$ & $20,9 \%$ \\
\hline Podemos & $20,7 \%$ & $21,4 \%$ \\
\hline IU & $3,7 \%$ & $9,3 \%$ \\
\hline Ciudadanos & $13,9 \%$ & $13,7 \%$ \\
\hline Upyd & $0,6 \%$ & $8,9 \%$ \\
\hline
\end{tabular}

Fuente: Elaboración propia.

No obstante, nuestra metodología para predecir los resultados y la tendencia por Twitter fracasa seriamente en el caso de IU y UPyD a quienes las redes sociales otorgan el 9,3\% y el 8,9\% de los votos y una representación segura en el Congreso que la formación magenta no llega siquiera a obtener (3,7\% y $0,6 \%$ de los votos). La desviación del método llega a los 5,5 puntos en el caso de Izquierda Unida y al 8,3 en UpyD (Ver Tabla 6).

\subsection{Análisis de contenido}

A nivel de contenido, corrupción, Empleo y Economía monopolizan el grueso de las conversaciones que los partidos mantienen en Twitter, seguido a gran distancia de temas referentes a Política, Sanidad, Sociedad y Educación.

Como puede apreciarse en la Tabla 7 , los resultados arrojan datos significativos: mientras que la Corrupción domina en el conjunto informativo de los partidos de izquierda y nuevas formaciones, el Empleo y la Economía son dos temas recurrentes en idéntica proporción en todas las formaciones, con la diferencia de que mientras 
el PP hace alusiones a la recuperación económica lograda durante su gobierno, el resto de formaciones articula sus mensajes para denunciar el fracaso de las políticas económicas del PP para reducir el paro y la exclusión social.

Por su parte, la política, centrada sobre todo en el debate territorial de Cataluña y en la defensa de la unidad de España, conforma el 20\% del discurso del PP; mientras que la Educación es un tema menor que preocupa, sobre todo a Ciudadanos (11\%) e Izquierda Unida (10\%).

Tabla 7. Contenido de los mensajes emitidos en Twitter.

\begin{tabular}{|l|c|c|c|c|c|c|}
\hline & Podemos & PP & PSOE & Ciudadanos & IU & UPyD \\
\hline Empleo & $20 \%$ & $21 \%$ & $25 \%$ & $18 \%$ & $14 \%$ & $21 \%$ \\
\hline Economía & $15 \%$ & $18 \%$ & $18 \%$ & $18 \%$ & $18 \%$ & $21 \%$ \\
\hline Sociedad & $8 \%$ & $11 \%$ & $6 \%$ & $6 \%$ & $5 \%$ & $8 \%$ \\
\hline Sanidad & $8 \%$ & $8 \%$ & $9 \%$ & $9 \%$ & $7 \%$ & $9 \%$ \\
\hline Política & $11 \%$ & $20 \%$ & $12 \%$ & $10 \%$ & $14 \%$ & $12 \%$ \\
\hline Corrupción & $30 \%$ & $8 \%$ & $18 \%$ & $23 \%$ & $28 \%$ & $19 \%$ \\
\hline Educación & $6 \%$ & $9 \%$ & $8 \%$ & $11 \%$ & $10 \%$ & $6 \%$ \\
\hline Otros & $2 \%$ & $5 \%$ & $4 \%$ & $5 \%$ & $4 \%$ & $4 \%$ \\
\hline
\end{tabular}

Fuente: Elaboración propia.

El contenido de los mensajes emitidos por las formaciones políticas durante la campaña electoral de 2015 en Twitter se corresponde con el resultado arrojado por el CIS, que revela que, al igual que testeaba la red social, el Paro $(79,4 \%)$, la Corrupción y el Fraude (55\%) y los problemas de índole económico $(24,5 \%)$ serían los temas más preocupantes para los españoles.

Además de la temática, a la hora de analizar cualitativamente los mensajes hemos aplicado un filtrado de mensajes que otorga un valor positivo, negativo o neutros a los tuits publicados y que nos indica de forma contundente que según lo expresado por los ciudadanos en esta red social los partidos con menos notoriedad (Podemos y Ciudadanos) continúan en progreso ascendente en cuanto a valoración y reputación, mientras que los tradicionales sufren el castigo del sistema actual.

El PP es la formación que acapara la mayor parte de las conversaciones $(40 \%)$, aunque en su mayoría en tono negativo, seguida de Podemos (27\%) y PSOE (19\%), al tiempo que la opinión ciudadana en Twitter confirma a Alberto Garzón (IU) y Albert Rivera (Ciudadanos) como los políticos mejor valorados y confirma el ascenso en estimación de voto directo de Podemos.

\section{Conclusiones}

Los datos obtenidos en esta investigación ponen de manifiesto que Twitter es una sonda permanente de opinión, y que a pesar de lo aleatorio de sus contenidos, bien administrados y organizados, proporciona un caudal público de información que supera con creces cualquier sondeo o muestreo, pues las opiniones espontáneas que se manifiestan reflejan fielmente el pulso de la sociedad. 
Sin embargo, el modelo propuesto no es válido en su totalidad, pues tan sólo ha resultado certero en el caso de las nuevas formaciones políticas que concurrían a las elecciones generales. El método empleado sólo llega a desviarse unas décimas en el caso de Podemos y Ciudadanos, superando, incluso, las previsiones realizadas por las encuestas de opinión tradicionales que mostraron desviaciones de hasta 5 puntos en el caso de ambas formaciones. Los datos obtenidos en este estudio también se acercan bastante al resultado obtenido por PP y PSOE, donde el estudio de la actividad comunicativa desarrollada en Twitter ofrece un error de 3,05 puntos porcentuales en el caso del PP y de 1,16 en el caso del PSOE.

El grado de acierto obtenido en el caso de las nuevas formaciones políticas que concurrían a estas elecciones generales pone de manifiesto que cuanto más jóvenes, ilusionados y motivados sean los seguidores, más activos se muestran a la hora de hacer visible sus siglas y sus opiniones, lo que hace de Twitter una herramienta bastante fiable a la hora de predecir los resultados electorales.

Sin embargo, esta herramienta de predicción electoral aporta resultados dispares en el caso de IU y UPyD, dándose un margen de error de hasta 8 puntos, lo que impide desarrollar un modelo válido. Las desviaciones obtenidas por ambas formaciones vienen motivadas, fundamentalmente, por el hecho de que la difusión de mensajes a través de Twitter es endógena, lo cual significa que son mensajes que interactúan principalmente con el mismo grupo de usuarios, de manera que sólo registran las ideas de aquellos que se expresan de forma digital, al tiempo que demuestran que hacerlo bien en las redes sociales no siempre es sinónimo de ganar votos.

Sin embargo, nuestro modelo de estudio no solo explora los datos cuantitativos que ofrecen un determinado tanto por ciento en intención de voto, sino que también revela datos cualitativos significativos que permiten mostrar cómo Twitter refleja la tendencia y el sentimiento político de la sociedad.

Pese a que las mediciones sociales todavía tienen ante sí un gran reto metodológico orientado a la capacidad de analizar y cuantificar aspectos muy concretos en lo que se refiere al modo de difusión del mensaje político, su alcance y el papel que juegan los diferentes tipos de usuarios; el estudio del flujo de mensajes y el sentimiento que de ellos se extrae nos ofrece datos que resultan ser un buen termómetro social y una información valiosa para complementar los datos de las encuestas, sobre todo cuando éstas han demostrado no ser muy fiables en caso de las formaciones políticas más jóvenes.

En este sentido, y como afirma Fernández Crespo (2013), se abre un campo de especial interés y relevancia de cara al futuro, en el que tal vez llegue a ser posible sustituir o convivir con las encuestas preelectorales, que hasta esta última cita electoral habían sido una fuente fiable de la tendencia electoral, al menos en los partidos consolidados que concurrían a las elecciones generales del 20-D. 


\section{Referencias bibliográficas}

Al-kandari, A. \& Hasanen, M. (2012). The impact of the Internet on political attitudes in Kuwait and Egypt. Telematics \& Informatics, 29(3), 245-253.

Alonso, M. (2015). Periodismo y Redes Sociales. La credibilidad en Twitter. Libro de Actas del IV Congresso Internacional de Ciberjornalismo, Oporto, 272-289.

Aparashivei, G. (2011). The Use of the New Media in Electoral Campaigns: Analysis on the Use of Blogs, Facebook, Twitter and YouTube in the 2009 Romanian Presidential Campaign. Journal of Media Research, 2(10), 39-60.

Barber, B. (2004). Which Technology and Which Democracy? En Jenkins, H. y Thorbur, D (eds.), Democracy and New Media (pp. 33-48). Cambridge: MIT Press.

Borondo, J., Morales, A.J., Losada, J.C. y Benito R.M. (2012). Characterizing and modeling an electoral campaign in the context of Twitter: 2011 Spanish Presidential Election as a Case Study. Chaos, 22.

Castells, M. (2009). Comunicación y Poder. Madrid: Alianza Editorial.

Cavaller, V.; Carreras, L.; Sánchez-Ã̃ón, S. y March, A. (2013). Medición y evaluación de la comunicación. Barcelona: UOC.

Chadwick, A. (2013). The hybrid media system: politcs and power. New York: Oxford University Press.

Creswel, J. \& Plano, V. (2011). Designing and Conducting Mixed Methods Research. Thousand Oaks: Sage Publications.

D’Adamo, O.; García, V. y Kievsky, T. (2015). Comunicación política y redes sociales: análisis de las campañas para las elecciones legislativas de 2013 en la ciudad de Buenos Aires. Revista Mexicana de Opinión Pública, julio - diciembre de 2015, 107-125.

Deltell, L.; Claes, F. y Osteso, J.M. (2013). Predicción de tendencia política por Twitter: Elecciones Andaluzas 2012. Ámbitos, 22. Recuperado de http://ambitoscomunicacion.com/2013/prediccion-de-tendencia-politica-por-Twitter -elecciones-andaluzas-2012/

Fernández Crespo, M. (2013). Predicción electoral mediante análisis de redes sociales. Memoria de Grado. Madrid: Universidad Complutense. Recuperado de http://eprints.ucm.es/22019/1/T34588.pdf

García, M.C., Del Hoyo, M. y Fernández, C. (2014). Jóvenes comprometidos en la Red: el papel de las redes sociales en la participación social activa. Comunicar, 43, 35-43.

García, A.; García, I. y Varona, D. (2012). Incidencia de las redes sociales vs. cibermedios en las Elecciones en España, 2011. Enl@ce Revista Venezolana de Información, Tecnología y Conocimiento, 9(2), 11-29.

García, C. y Zugasti, R. (2014). La campaña virtual en Twitter: análisis de las cuentas de Rajoy y Rubalcaba en las Elecciones Generales de 2011. Historia y Comunicación Social, 9, 299-311.

Gayo-Avello, D. (2011). Limits of Electoral Predictions Using Twitter. Association for the Advancement of Artificial Intelligence. Proceedings of the Fifth International AAAI Conference on Weblogs and Social Media, 490-493. Recuperado de http://www.aaai.org/ocs/index.php/ICWSM/ICWSM11/paper/view/2862.

Giansante, G. (2015). La comunicación política online. Barcelona: Editorial UOC. 
González, V. \& Petersen, M.D. (2010). The Reach of Twitter as a Political Tool. Orbis, Revista Científica Electrónica de Ciencias Humanas, 16(5), 98-116.

Herrero, J. (2014). Comunicación en campaña: dirección de campañas electorales y marketing político. Madrid: Pearson.

Jungherr, A.; Jürgens, P. y Schoen, H. (2012). Why the Pirate Party Won the German Election of 2009 or The Trouble With Predictions: A Response to Tumasjan et.al.: Predicting Elections With Twitter: What 140 Characters Reveal About Political Sentiment. Social Science Computer Review, 30(2), 229-234.

Larsson, A. \& Moe, H. (2010). Studying political microblogging: Twitter users in the 2010 Swedish election campaign. New Media \& Society, 14 (5), 729-747.

Leiva-Aguilera, J. (2012). Gestión de la reputación online. Barcelona: UOC.

Lopez-García, G. (2016). Nuevos' y 'viejos' liderazgos: la campaña de las elecciones generales españolas de 2015 en Twitter. Communication \& Society, 29(3), 149-167.

Maarek, P. (2014). Politics 2.0. New Forms of Digital Political Marketing and Political Communication. Trípodos, 34, 13-22.

Martins, T. y Azevedo, I. (2015). Tendências no Twitter. Um Estudo de Caso. En Rocha, A.; Martins, A.; Paiva, G.; L.P. Reis, L.P. y Pérez, M. (Eds.), Sistemas e Tecnologias de Informação. Atas da 10a . Conferência Ibérica de Sistemas e Tecnologias de Informação (pp. 922-927). Águeda: AISTI. Disponible en: http:// www.aisti.eu/cisti2015/index.php/es/proceedings

Monge, J. J. (2011). La agenda setting o cómo los medios manejan la información, en Qué aprendemos hoy. Recuperado de http://queaprendemoshoy.com/ la-agenda-setting-o-como-los-medios-manejan-la-informacion/

Mutz, D. (2002). Cross-cutting social networks: Testing democratic theory in practice. American Political Science Review, 96, 111-126.

O’Connor, B.; Balasubramanyan, R.; Routledge, B. \& Smith, N. (2010). From tuits to polls: Linking text sentiment to public opinion time series. Proc. of 4th ICWSM, pp. 122-129. AAAI Press.

Panagiotis, E. (2011). How (not) to Predict Elections. IEEE Third International Conference on Social Computing (pp. 165-171). Recuperado de http://www.academia.edu/2232800/How_not_to_predict_elections

Pérez-Dasilva, J.; Santos, T. y Meso K. (2015). Radio y redes sociales: el caso de los programas deportivos en Twitter. Revista Latina de Comunicación Social, 70, 141-155.

Resina, J. (2010). Ciberpolítica, redes sociales y nuevas movilizaciones en España: el impacto digital en los procesos. Revista Mediaciones Sociales, 10, 143-164.

Rodríguez, R.A. y Ureña, D. (2011). Diez razones para el uso de Twitter como herramienta de comunicación política y electoral. Comunicación y Pluralismo, 10, 89-116.

Rojas, H. (2006). Comunicación, participación y democracia. Universitas Humanística, 62, 109-142.

Ros-Martin,M.(2005).Lablogosferacomonoosfera(oideosfera).Recuperadodehttp:// www.documentalistaenredado.net/180/la-blogosfera-como-noosfera-o-ideosfera.

Rúas-Araujo, J.; Puentes-Rivera, I; Míguez-González, M.I. (2016). Capacidad predictiva de Twitter, impacto electoral y actividad en las elecciones al Parlamento 
de Galicia: un análisis con la herramienta LIWC. Observatorio Journal, 10(2), 55-87.

Said, E. \& Arcila, C. (2011). Los líderes de opinión en Colombia, Venezuela e Irán. El caso de los 20 usuarios más vistos en Twitter. Comunicación y Sociedad, 24(1), 75-100.

Semetko, H. \& Valkeburg, P. (1998). The impact of attentiveness on political efficacy: Evidence from a three-year German panel study International Journal of Public Opinion Research, 10, 195-210.

Shah, D.; Cho, J.; Eveland, W. \& Kwak, N. (2005). Information and Expression in a Digital Age Modeling Internet Effects on Civic Participation. Communication Research, 28, 464-506.

Sunstein, C. (2009). On rumours. How Falsehoods Spread, Why We Believe Them, What Can Be Done. Penguin: Londres.

Tjong, E. y Bos, J. (2012). Predicting The 2011 Dutchd Senate Election Results With Twitter. Association for Computational Linguistics. Proceedings of the 13th Conference of the European Chapter of the Association for Computational Linguistics, 53-60.

Tumasjan, A.; Sprenger, T.; \& Welp, I. (2012). Predicting Election with Twitter: What 140 Characters Reveal about Political Sentimen. Social Science Computer Review, 30, 229-234.

Tweetminster (2010). Is word-of-mouth correlated to General Election results? The results are in. Recuperado de http://es.scribd.com/doc/31208748/ Tweetminster-Predicts-Findings

Véronis, J. (2007). La presse a fait mieux que les sondeurs. Recuperado de http:// aixtal.blogspot.com/2007/04/2007- la-presse-fait-mieux-que-les.html.

Wilson, J. (2011). Playing with politics: Political fans and Twitter faking in postbroadcast democracy. Convergence, 17(4), 445-461.

Zamora, R. y Zurutuza, C. (2014). Campaingning on Twitter: Towards the 'Personal Style' Campaging to Activate the political Engagenment during the 2011 Spanish General Elections. Communication y Society, 27(1), 87-106.

Zugasti, R. y Sabés, F. (2015). Los issues de los candidatos en Twitter durante la campaña de las elecciones generales de 2011. ZER Revista de Estudios de Comunicación, 20(38), 161-178. 